\title{
Review
}

Basic Research

Diabetes Metab J 2021;45:840-852

https://doi.org/10.4093/dmj.2020.0291

pISSN 2233-6079 · eISSN 2233-6087

DIABET\&S \& METABOLISM JOURNAL

\section{Brown Fat as a Regulator of Systemic Metabolism beyond Thermogenesis}

\author{
Okamatsu-Ogura Yuko ${ }^{1}$, Masayuki Saito ${ }^{1,2}$ \\ ${ }^{1}$ Faculty of Veterinary Medicine, Hokkaido University, Sapporo, \\ ${ }^{2}$ Department of Nutrition, Tenshi College, Sapporo, Japan
}

Brown adipose tissue (BAT) is a specialized tissue for nonshivering thermogenesis to dissipate energy as heat. Although BAT research has long been limited mostly in small rodents, the rediscovery of metabolically active BAT in adult humans has dramatically promoted the translational studies on BAT in health and diseases. Moreover, several remarkable advancements have been made in brown fat biology over the past decade: The molecular and functional analyses of inducible thermogenic adipocytes (socalled beige adipocytes) arising from a developmentally different lineage from classical brown adipocytes have been accelerated. In addition to a well-established thermogenic activity of uncoupling protein 1 (UCP1), several alternative thermogenic mechanisms have been discovered, particularly in beige adipocytes. It has become clear that BAT influences other peripheral tissues and controls their functions and systemic homeostasis of energy and metabolic substrates, suggesting BAT as a metabolic regulator, other than for thermogenesis. This notion is supported by discovering that various paracrine and endocrine factors are secreted from BAT. We review the current understanding of BAT pathophysiology, particularly focusing on its role as a metabolic regulator in small rodents and also in humans.

Keywords: Adipocytes, beige; Adipose tissue; Adipose tissue, brown; Energy metabolism; Thermogenesis; Uncoupling protein 1

\section{INTRODUCTION}

Mammals have two types of adipose tissue, the white adipose tissue (WAT) and brown adipose tissue (BAT), which have opposite physiological roles: WAT is the site to store energy as triglyceride (TG), whereas BAT is a specialized tissue for nonshivering thermogenesis (NST) to dissipate energy as heat [1]. Although BAT research has long been limited mostly to small rodents, the rediscovery of metabolically active BAT using radionuclide imaging technique in adult humans [2-5] has dramatically accelerated the translational studies on BAT in health and diseases, particularly on its role in the regulation of energy balance, body fatness, and substrate metabolism [6,7]. Over the past decade, several remarkable advancements have been made in the field of brown fat biology. First, increasing evi- dence has been accumulated indicating rodents and humans possess two types of thermogenic adipocytes arising from developmentally distinct lineages: the classical brown adipocytes and the so-called beige or brite cells that are inducible within WAT [8]. Second, in addition to a well-established thermogenic activity of uncoupling protein 1 (UCP1), several alternative molecular mechanisms of NST have been discovered, particularly in beige adipocytes [9]. Third, studies in rodents and humans have revealed that BAT cross talks with some peripheral tissues and controls their functions, systemic homeostasis of energy, and metabolic substrates, suggesting BAT as a metabolic regulator beyond thermogenesis [10]. In fact, various paracrine and endocrine factors are found to be secreted from BAT [11]. In this review, we summarize our current understanding on BAT pathophysiology, particularly focusing on its
Corresponding author: Masayuki Saito (D) https://orcid.org/0000-0002-3058-3003 Faculty of Veterinary Medicine, Hokkaido University, Sapporo 060-0818, Japan

E-mail: saito@vetmed.hokudai.ac.jp

Received: Dec. 8, 2020; Accepted: Jan. 26, 2021
This is an Open Access article distributed under the terms of the Creative Commons Attribution Non-Commercial License (https://creativecommons.org/licenses/by-nc/4.0/) which permits unrestricted non-commercial use, distribution, and reproduction in any medium, provided the original work is properly cited. 
role as a metabolic regulator in small rodents and humans.

\section{UCP-DEPENDENT AND -INDEPENDENT MECHANISMS OF BAT THERMOGENESIS}

The most well-established thermogenic molecule for NST, particularly for cold-induced thermogenesis (CIT), is UCP1 expressed exclusively in BAT. UCP1 has the ability to leak proton gradient across the mitochondrial membrane, and thereby uncouples oxidative phosphorylation to generate heat. When animals are exposed to the cold, temperature sensors (transient receptors potentially channel) on sensory neurons on the body surface transmit information to the brain and increase the activity of sympathetic nerves entering BAT. Noradrenaline released from sympathetic nerve endings stimulates BAT via $\beta$-adrenergic receptor ( $\beta A R)$ and triggers cyclic adenosine monophosphate-activated intracellular events, including TG hydrolysis, fatty acid (FA) oxidation, and UCP1 activation (Fig. 1).
The critical role of UCP1 in CIT is typically indicated in mice with genetic deletion of UCP1 (UCP1-knockout [KO]), making them cold-sensitive and unable to maintain their body temperature after acute cold exposure [12]. However, it is also true that UCP1-KO mice can survive during mild and gradual cold exposure, suggesting some thermogenic mechanisms other than UCP1 $[13,14]$. In general, when animals are exposed to cold temperatures for a long time, they adapt to their surroundings by increasing the number of brown adipocytes and the amount of UCP1 through the proliferation of interstitial preadipocytes and mature adipocytes $[15,16]$. In addition to BAT hyperplasia, prolonged cold exposure increases an apparent induction of beige adipocyte in WAT. Beige adipocyte expresses considerable amounts of UCP1 and has thermogenic activity comparable with that of brown adipocyte $[17,18]$. However, recent studies have indicated the existence of UCP1independent thermogenic mechanisms, particularly in beige adipocytes (Fig. 1).

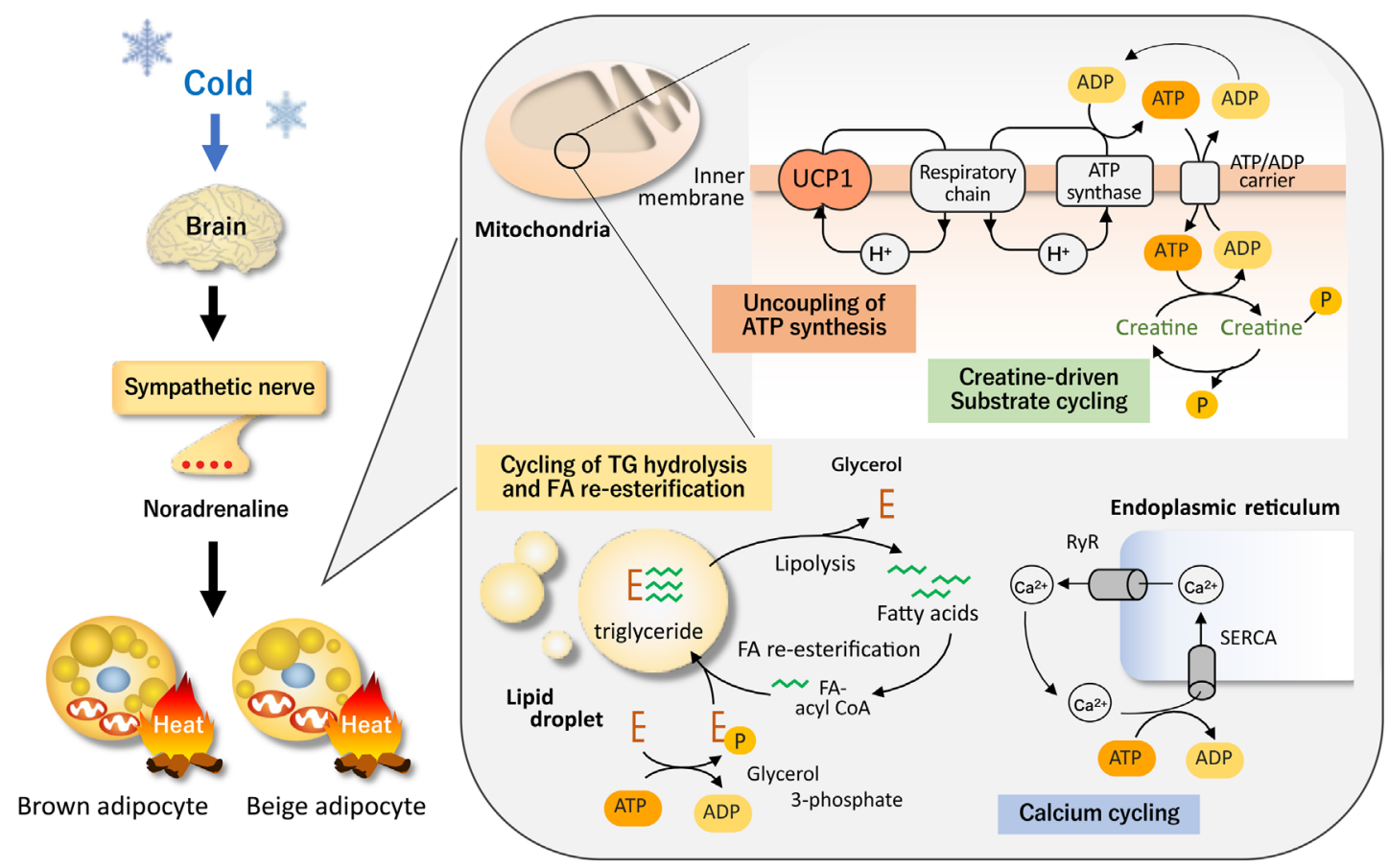

Fig. 1. Uncoupling protein 1 (UCP1)-dependent and independent thermogenesis in brown and beige adipocytes. UCP1, a protein located in the mitochondrial inner membrane, has the ability to leak proton gradient across the mitochondrial membrane, and thereby uncouples oxidative phosphorylation to generate heat. Thus, UCP1 thermogenesis is not dependent on adenosine triphosphate (ATP). On the other hand, thermogenesis by the futile cycles of creatine and creatine phosphate, release and re-uptake of calcium, and triglyceride (TG) hydrolysis and fatty acid (FA) re-esterification is dependent on the breakdown of ATP to adenosine diphosphate (ADP). These thermogenic mechanisms are activated by cold exposure primarily through the sympathetic nerve activation. RyR, ryanodine receptor; SERCA, sarco-endoplasmic reticulum ATPase. 
Beige adipocytes contain large amounts of mitochondria and a high capacity of oxidative phosphorylation for adenosine triphosphate (ATP) synthesis. Thus, ATP-dependent futile cycles can compensate for the lack of UCP1 thermogenesis. Calcium cycling is one of the ATP-dependent and UCP1-independent futile mechanisms. Calcium transport has been known to contribute to NST in muscles through sarco-endoplasmic reticulum ATPase (SERCA) and ryanodine receptors (RyR) [19]. Ikeda et al. [20] demonstrated that this ATP-dependent calcium cycling also occurs through SERCA2b and RyR2 in beige adipocytes. Notably, the SERCA2b-mediated $\mathrm{Ca}^{2+} \mathrm{cy}-$ cling mechanism is necessary for beige adipocyte thermogenesis, but dispensable in brown adipocytes.

Another example of UCP1-independent thermogenic mechanisms is creatine cycling through ATP-dependent phosphorylation and dephosphorylation [21]. Cycling of FA and TG through hydrolysis of TG and re-esterification of released FA is also possible as an ATP-dependent thermogenic mechanism in beige adipocytes [22]. Thus, all of these UCP1-independent thermogenic mechanisms would compensate for the lack of UCP1 and enable cold tolerance in UCP1-KO mice.

In adult humans, BAT is usually assessed by cold-activated glucose utilization using ${ }^{18} \mathrm{~F}$-fluorodeoxyglucose (FDG)-positron emission tomography (PET) [2-6]; that is, when FDG$\mathrm{PET}$ is performed after either cold exposure or $\beta 3$-AR agonist administration, it detects symmetrical FDG uptake in adipose tissue at the supraclavicular and thoracic spine regions. Histological examinations revealed the presence of UCP1-positive adipocytes surrounded by numerous UCP1-negative white adipocytes in these regions. Gene expression analysis has demonstrated that the fat pat at these regions is largely composed of beige adipocyte more than classical brown adipocytes [23,24], suggesting both UCP1-dependent and -independent thermogenic mechanisms in adult humans; however, their individual roles remain to be elucidated.

\section{THERMOGENESIS-ASSOCIATED METABOLIC CHANGES IN BAT}

As noted above, the primary energy source of BAT thermogenesis is FA. FA is also indispensable for BAT thermogenesis as an activator of UCP1 [25-28]. Thus, BAT thermogenesis seems intimately associated with intracellular TG hydrolysis and FA oxidation. On the other hand, there have been reports that incorporation of FA from circulation and its synthesis in
BAT are also enhanced after cold exposure and/or sympathetic stimulation [29-31]. As such, lipid metabolism is drastically changed in parallel with activation of BAT thermogenesis; however, their causal relation to UCP1-dependent thermogenesis is not fully understood.

Recently, we conducted metabolomic and GeneChip array analyses of BAT after acute cold exposure in wild-type (WT) and UCP1-KO mice [32]. Our results suggest that the FA uptake, oxidation, and intracellular lipolysis simultaneously occur after cold exposure in WT and UCP1-KO mice, indicating these changes are UCP1-independently induced probably through the sympathetic nerve activation. On the other hand, FA-derived acetyl-coenzyme A ( $\mathrm{CoA})$ is oxidized through the tricarboxylic acid (TCA) cycle and oxidative phosphorylation in WT mice, while it is converted to citric acid and utilized for active de novo FA synthesis in UCP1-KO mice (Fig. 2). We also found that succinate, an intermediate of the TCA cycle, was accumulated after cold exposure in BAT of WT, but not in UCP1$\mathrm{KO}$ mice. This is consistent with the reports that succinate is incorporated into brown adipocytes upon cold exposure and activates thermogenesis by producing reactive oxygen species [33], which activate thermogenesis through UCP1 sulfenylation [34].

In addition to lipid, glucose metabolism is also drastically changed in parallel with the activation of BAT thermogenesis. Cold exposure has been known to increase 2-deoxy-D-glucose (2-DG) uptake into BAT but only slightly into WAT $[35,36]$. The stimulatory effects of cold exposure can be mimicked by electrical stimulation of sympathetic nerves into BAT and $\beta$-adrenergic agonist administration, but are abolished by either sympathetic nerve surgery, $\beta$-adrenergic blockade, or genetic deletion of UCP1 $[37,38]$. Thus, the $\beta$-adrenergically stimulated 2-DG uptake into BAT is dependent on UCP1-mediated thermogenesis. Moreover, our metabolomic analysis revealed that glycolytic metabolites in BAT robustly increased by cold exposure in WT mice, but not in UCP1-KO mice [32]. In addition, lactic acid levels elevated only in WT mice, suggesting that a large proportion of glucose incorporated in BAT is metabolized via anaerobic glycolysis. It is most likely that glucose is used to replenish ATP through aerobic glycolysis to compensate for the decreased ATP production in mitochondria caused by UCP1 uncoupling. Thus, cold-induced increase in glucose utilization in BAT is secondary to UCP1 thermogenesis. This is a basis to use FDG-PET for the assessment of human BAT. It was also reported that inhibition of glycolysis 


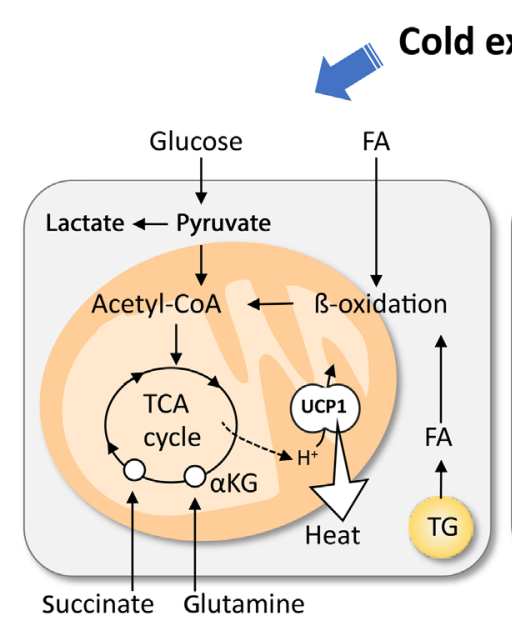

Wild-type mice

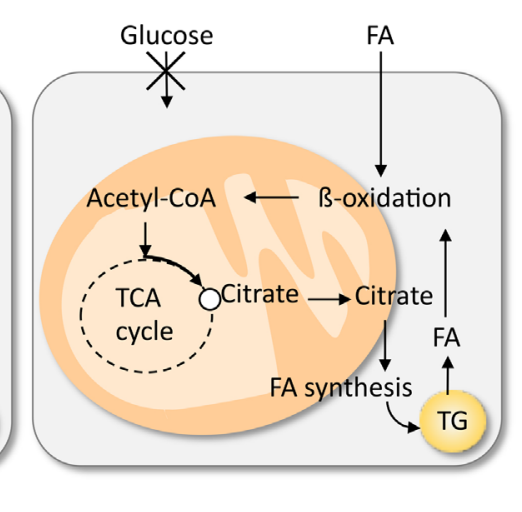

UCP1-KO mice

Fig. 2. Cod-induced uncoupling protein 1 (UCP1)-dependent and -independent metabolic changes in brown adipose tissue (BAT). Cold exposure triggers triglyceride (TG) hydrolysis, fatty acid (FA) uptake, oxidation of FA via $\beta$-oxidation and tricarboxylic acid (TCA) cycle, and UCP1 activation. These are intimately associated with anaerobic glycolysis, and uptake of succinate and glutamine. In UCP1-KO brown adipocytes, cold exposure triggers TG hydrolysis, FA uptake, and $\beta$-oxidation of FA, similarly in wild-type brown adipocytes. Resulting acetyl-coenzyme A (CoA) is not oxidized by TCA cycle but used to re-synthesis of FA and TG. aKG, a-ketoglutaric acid.

impairs BAT thermogenesis [39,40]. This may contradict with the above-mentioned idea but may easily be explained by assuming that increased glycolysis is favorable and indispensable for a sufficient supply of oxaloacetate to enable rapid oxidation of FA and acetyl-CoA by the TCA cycle.

Some studies demonstrated an apparent association of amino acid metabolism and thermogenesis in BAT [41-43]. For example, acute cold exposure in rats increased BAT influx of amino acids, including glutamine and branched-chain amino acids (BCAAs), accounting for approximately one-third of the energy supplied by glucose to the tissue [44]. Indeed, glutamine level increased upon cold exposure in WT mice, but not in UCP1-KO mice [32]. Adipose tissue is known to have active glutamine metabolism [43] and contains a high level of glutaminase, an enzyme responsible for the conversion of glutamine to glutamate, reaching approximately $33 \%$ of the entire enzyme activity in rat lymphocytes or kidney [45]. Cold-induced uptake of glutamine is possibly related to the UCP1-thermogenesis induced tissue hypoxia [46], which is reported to enhance tissue uptake of glutamine used for citrate production from $\alpha$-ketoglutaric acid [47] or de novo FA synthesis [48]. BCAA is also actively used as a substrate for thermogenesis and promotes systemic BCAA clearance. Recently, Yoneshiro et al. [49] demonstrated that BAT highly expresses the enzymes for
BCAA catabolism and actively imports BCAA into mitochondria via the specific transporter SLC25A44.

\section{BAT AS A REGULATOR OF SYSTEMIC METABOLISM}

BAT thermogenesis is associated with metabolic changes in BAT itself and those in a wide range of systemic metabolism. The most representative is the regulatory role of whole-body energy expenditure (EE) and body fatness. Activation of BAT thermogenesis by cold exposure or $\beta A R$ stimulation leads to an increase in whole-body EE and a concomitant decrease in WAT fat content. In diet-induced or genetically obese animals, whole-body EE and BAT activity are reduced, but BAT transplantation increases whole-body EE and reverses obesity [50,51]. The significant role of BAT in body fat regulation appears controversial because UCP1-KO mice are cold-sensitive but not obese $[52,53]$. This paradoxical finding was re-examined by Kontani et al. [54], who demonstrated that UCP1-KO mice get obese when they are kept on a high-fat diet for more than half a year. Feldmann et al. [55] also reported that UCP1$\mathrm{KO}$ are susceptible to diet-induced obesity when they are maintained at thermoneutral temperatures $\left(29^{\circ} \mathrm{C}\right)$, but not at conventional animal room temperatures $\left(18^{\circ} \mathrm{C}\right.$ to $\left.22^{\circ} \mathrm{C}\right)$. Thus, 
the preventive effect of UCP1 against body fat accumulation is actualized only when animals need not to increase CIT. Therefore, in the absence of UCP1, some alternative calorically more costly mechanisms are used for thermogenesis to maintain body temperature, which also contribute to body fat regulation. The ATP-dependent futile cycles may be a likely mechanism. In fact, the calcium cycling mechanism activated by sarcolipin is upregulated in the skeletal muscle of UCP1-KO mice, and UCP1 expression is increased in sarcolipin-KO mice [14].

As noted in section 2, the main energy source of BAT thermogenesis is FA derived from intracellular TG and incorporated from circulation. BAT activation by cold exposure drastically accelerates clearance of plasma TG-rich lipoproteins (TRL) due to increased uptake into BAT. This depends on lipoprotein lipase and a lipid transporter CD36, and corrects hyperlipidemia in Apoa5-KO mice [56]. Similar effects were also found by pharmacologic activation of BAT with $\beta 3 \mathrm{AR}$ agonist CL316,243, which improves dyslipidemia, and more importantly, protects hyperlipidemic model mice from atherosclerosis [57]. Activation of BAT thermogenesis increases high-density lipoprotein (HDL) levels, promoting HDL cholesterol turnover [58], which may also contribute to the atheroprotective effects of BAT. All these facts suggest a direct contribution of BAT to the regulation of blood lipoprotein metabolism.

The significant contribution of BAT to blood metabolite levels was also documented in BCAA; that is, BCAA uptake into activated BAT produces an apparent reduction of plasma BCAA levels [49], which may improve insulin sensitivity in other peripheral tissues as noted in section 5 .

Possible roles of BAT in whole-body glucose homeostasis have been suggested over the years. It has been reported that cold exposure potentiates the stimulatory effect of insulin and increases glucose uptake in peripheral tissues, including skeletal muscle, WAT, and BAT, resulting in improved glucose tolerance [59]. Direct evidence for the beneficial effects of BAT on glucose homeostasis has been accumulated in studies of BAT transplantation. Gunawardana and Piston [60] first demonstrated that subcutaneous transplants of embryonic BAT can correct type 1 diabetes mellitus in streptozotocin-treated mice with severely impaired glucose tolerance. In support of their observation, transplantation of BAT was shown to result in improved glucose tolerance, increased insulin sensitivity, lower body weight, decreased fat mass, and a complete reversal of high-fat diet-induced insulin resistance [61]. Inversely, surgical removal of the interscapular BAT in obese diabetic mice im- pairs insulin sensitivity [62]. Moreover, Nishio et al. [63] reported that mice with transplanted brown adipocytes, derived from human pluripotent stem cells (hPSC), show augmented glucose tolerance, while those with hPSC-derived white adipocytes show deteriorated glucose tolerance. Notably, co-transplantation of brown adipocytes normalizes glucose intolerance induced by white adipocyte transplantation. There have also been reports that induction of beige adipocytes by transgenic techniques improves glucose tolerance in $\mathrm{WT}$ and even in UCP1-KO mice [20], indicating that UCP1 is not necessarily indispensable for the beneficial effects of brown and beige adipocytes on glucose homeostasis.

\section{BAT-DERIVED ENDOCRINE FACTORS REGULATING SYSTEMIC METABOLISM}

As discussed above, even small amounts of BAT influence the broad range of systemic metabolisms, suggesting the role of BAT as a regulator of metabolism beyond thermogenesis. Mechanistically, some of the effects may be attributed to the relatively high metabolic activity of BAT itself, as in the case of the lowering effect on TRL and BCAA; however, those on glucose metabolism and insulin sensitivity may not be explained by this activity. In this connection, it is interesting that BAT secretes various molecules into extracellular fluid, collectively called as BATkines, which may mediate the effects of BAT on other tissues (Fig. 3) [11].

It is known that BAT and WAT synthesize and secrete various adipokines, including adiponectin. In addition, activated BAT secretes some growth factors, such as vascular endothelial growth factor and nerve growth factors, which promote angiogenesis and sympathetic nerve growth associated with tissue hyperplasia [64-66]. In addition to these paracrine factors, BAT secretes a wide variety of molecules into circulating blood, including polypeptides, FA metabolites, and microRNA (miRNA).

Interleukin 6 (IL-6) is one of the polypeptides secreted from BAT. In the study of BAT transplantation mentioned in section 3, Stanford et al. [61] found that the improved metabolic profile was lost when the BAT used for transplantation was obtained from IL-6-KO mice, demonstrating that BAT-derived IL-6 is required for the profound effects of BAT transplantation on glucose homeostasis and insulin sensitivity. Recently, Qing et al. [67] reported that acute stress induces IL-6 secretion from BAT, which is the required instructive signal for me- 


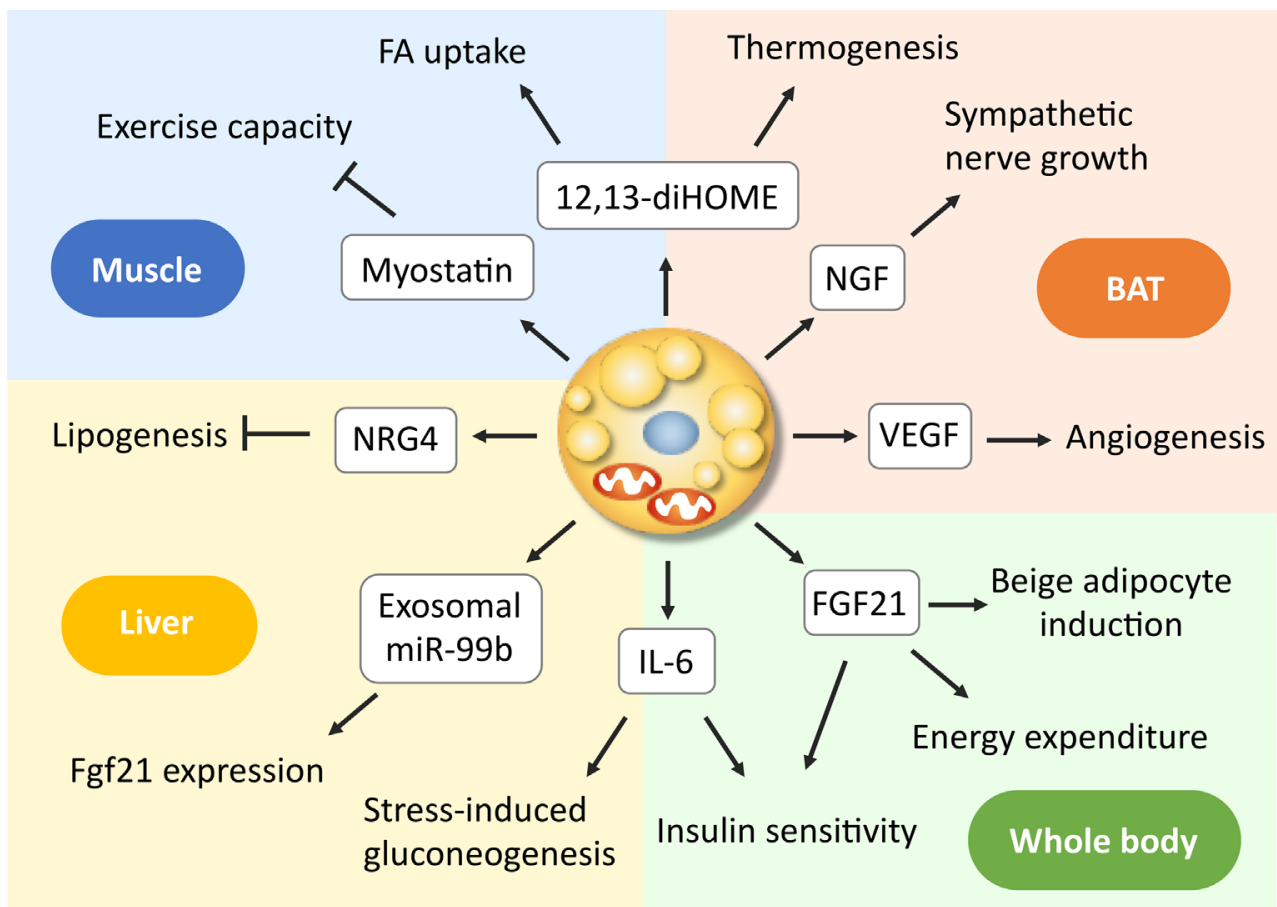

Fig. 3. Endocrine actions of brown adipose tissue (BAT)-derived factors. Brown/beige adipocytes secrete various molecules, some of which such as nerve growth factor (NGF) and vascular endothelial growth factor (VEGF) function as paracrine factors, while others as endocrine factors, acting on the liver and skeletal muscle, thereby regulating systemic metabolism. 12,13-diHOME, 12,13-dihydroxyoctadecaenoic acid; FA, fatty acid; FGF21, fibroblast growth factor 21; IL-6, interleukin-6; miR, microRNA; NRG4, neuregulin 4 .

diating hyperglycemia through hepatic gluconeogenesis. Thus, IL-6 may be a likely BATkine to regulate systemic glucose metabolism. Interestingly, the secretion of IL- 6 from BAT is mediated by $\beta 3 A R$ but independently of UCP1 thermogenesis.

Another candidate of BATkines is fibroblast growth factor 21 (FGF21). FGF21 is an important regulator of insulin sensitivity, systemic metabolism, and EE [68]. Although FGF21 is released abundantly from the liver, it is also secreted from BAT in response to cold exposure or $\beta 3 \mathrm{AR}$ stimulation and contributes to increased circulating levels in mice and humans [6971]. Some studies have demonstrated that UCP1 is dispensable for the action of FGF21 on body weight and glucose metabolism [72,73]. In contrast, FGF21 induces beige adipocyte biogenesis [74], suggesting that the FGF21 action is to stimulate some UCP1-independent mechanisms in beige adipocytes. In fact, Keipert et al. [75] reported that FGF-signaling controls paradoxical obesity resistance of UCP1-KO mice. Ruan et al. [76] also reported that brown adipocyte-specific FGF21 KO impaired the observed effects of adenosine A2A receptor agonism in attenuating hypertensive cardiac remodeling, suggest- ing an endocrine role of BAT in controlling hypertensive cardiac remodeling though the release of FGF21.

miRNAs are small noncoding RNAs with 20 to 22 nucleotides, that function as negative regulators of protein synthesis at the translational level and are involved in many cellular processes. miRNAs produced in adipocytes have a role in the differentiation and function of adipocyte itself and are also secreted through exosomes and taken-up into other cells. Thomou et al. [77] reported that mice with adipose-tissue-specific $\mathrm{KO}$ of the miRNA-processing enzyme exhibit a substantial decrease in the levels of circulating exosomal miRNAs, including miRNA-99b, and that the transplantation of BAT into the KO mice restores the circulating miRNA level, improves glucose tolerance, and suppresses hepatic FGF21 expression. These results suggest that exosomal miRNA-99b is secreted from BAT into blood circulation, acts on the liver, and suppresses hepatic FGF21 expression. It was also reported that circulating levels of miR-92a-3p and miRNA-122 are inversely correlated with BAT activity in humans $[78,79]$. Thus, it is likely that miRNA is a group of BATkines, although its precise role as a BATkine is 
largely elusive to date.

Lipid molecules also serve as signals between BAT and other tissues. When FA derived from cold-induced lipolysis in WAT is oxidized in liver and in BAT, the acylcarnitine formed from carnitine and acyl-CoA by carnitine acyltransferases are secreted into circulation, and incorporated into BAT to be utilized as energy fuel for thermogenesis [80]. Oxylipins, molecules derived from the oxidation of polyunsaturated FAs, are attracted interest because of their action as intercellular signaling molecules and involvement in the regulation of many cell and tissue responses. 12,13-Dihydroxyoctadecaenoic acid (12,13-diHOME) is secreted from BAT upon cold exposure and activates BAT in paracrine manner to enhance thermogenesis, resulting in decreased levels of serum TGs [81]. 12,13-diHOME secreted from BAT is also enhanced by exercise and increases FA uptake in muscle [82].

In addition, many molecules such as neureglin 4 (NRG4) [83] and myostatin [84] were identified as BATkines, but a comprehensive overview is beyond the scope of this article and found in other literatures $[11,66,85,86]$.

Besides these BATkines, afferent nerve may also be involved in the signaling from BAT. We found that chronic $\beta 3 A R$ agonist treatment potentiates anorexigenic effects of leptin at the hypothalamus level, in parallel with UCP1 induction in WAT, in WT mice, but not in UCP1-KO mice [87]. Yamada et al. [88] also showed that ectopic expression of very low levels of UCP1 in epididymal WAT improves glucose tolerance and reverses both insulin and leptin resistance in obese mouse models. Interestingly, local-nerve dissection in the epididymis or pharmacological afferent blockade blunts the leptin action, suggesting that afferent-nerve signals from intra-abdominal fat tissue regulate food intake by modulating hypothalamic leptin sensitivity. Histological and physiological evidence has indicated that BAT has sensory system innervation overlapping with the sympathetic efferent system [89]. Thus, it may be possible, that the BAT-derived sensory nervous system, in addition to and in combination with BATkines, plays a role in the regulation of systemic metabolism through the central nervous system.

\section{BAT IN HUMAN PHYSIOLOGY AND DISEASE}

Although our understanding on BAT discussed above is based largely on the studies in small rodents, the rediscovery of meta- bolically active BAT using FDG-PET in adult humans [2-5] has dramatically accelerated the translational studies on BAT in health and diseases.

A role of BAT to NST in humans has been demonstrated for CIT and diet-induced thermogenesis (DIT); that is, wholebody EE after mild cold exposure or meal intake is greater in individuals with higher BAT activities than those with lower activities [7,90-95]. This is also supported by the observations that a single nucleotide substitution in the UCP1 gene at -3826A to G, which lowers UCP1 mRNA expression [96], attenuates both CIT and DIT [97,98]. Muzik et al. [99] reported that CIT estimated from whole-body EE is much smaller than EE by BAT directly estimated by ${ }^{15} \mathrm{O}_{2}$-PET, contradicting a significant role of BAT detected by FDG-PET in whole-body EE and CIT. This discrepant result may be caused by FDG-PET which underestimated the total mass of BAT [100]. Consistent with the role of BAT in short-term regulation of EE, BAT is thought as a long-term regulator of energy and body fats in humans. Both clinical and experimental studies have revealed an inverse relationship between the activity/ prevalence of BAT and adiposity-related parameters, such as body mass index, body fat content, and visceral fat accumulation [101-104]. Moreover, cold-induced reactivation and recruitment of BAT in individuals with low BAT activities results in a significant increase in CIT and a concomitant reduction of body fat content [105-107].

Accumulated evidence has suggested a regulatory role of BAT in glucose homeostasis and insulin sensitivity in humans. We found in healthy adults that blood glucose and glycosylated hemoglobin (HbAlc) levels are lower in individuals with higher BAT activities [104]. Regression analysis revealed that BAT, in addition to age, sex, and body fatness, is a significantly independent determinant of glucose and HbAlc. These results seem compatible with some retrospective analyses of patient data showing blood glucose as a determinant of BAT prevalence $[4,102,103]$. Chondronikola et al. [108] reported that mild cold exposure for 5 hours increased whole-body glucose disposal, plasma glucose oxidation, and insulin sensitivity in men with significant amounts of BAT, but not in those without detectable BAT. Improved insulin sensitivity in parallel with BAT recruitment after cold acclimation was also shown in healthy men $[109,110]$ and in patients with type 2 diabetes mellitus [111].

In connection with the beneficial effects of BAT on insulin sensitivity, BAT also actively metabolizes BCAA to lower their 
plasma levels in humans [49]. Accordingly, impaired BAT activity reduces BCAA clearance, thereby resulting in increased circulating BCAA levels, which are often seen in obesity and aging [112]. The accumulation of intracellular BCAA results in mammalian target of rapamycin activation and subsequent inhibition of insulin signaling [113].

Very recent studies also revealed significant beneficial effects of BAT on the cardiovascular system in humans, in agreement with the atheroprotective effects of BAT in mice [57]. Becher et al. [114] categorized 53,475 patients by presence or absence of BAT, and found improved profiles of blood glucose, TG, and HDL-cholesterol in individuals with BAT, as already reported in healthy humans. Notably, BAT independently correlated with lower rates of hypertension, type 2 diabetes mellitus, coronary artery disease, and congestive heart failure. These results are well consistent with a 5-year follow-up study reported by Raiko et al. [115] showing that BAT activity correlated with lower carotid intima-media thickness and higher carotid elasticity.

\section{CONCLUSIONS}

As discussed in the previous sections, the preclinical and clinical studies, together with experimental studies in mice, have indicated that BAT is important as a regulator of whole-body energy balance, systemic metabolism, and cardiovascular functions. Hence, BAT is now recognized as an intriguing therapeutic target of obesity and metabolic disorders such as diabetes mellitus, dyslipidemia, and related cardiovascular diseases. In fact, several drugs and food ingredients targeting BAT have been tested for pharmacological and nutritional therapy of obesity and metabolic syndrome [116,117].

Despite these advances in experimental and clinical researches of BAT, some important questions remain to be solved: for example, in mouse models, beige fat induction is greatly influenced by their genetic background [118,119], but in humans little is known about possible effects of genetic variants on brown and beige adipocytes, which undoubtedly participate in the remarkable individual difference in the amount and activity of BAT [120]. Another problem is related to the method used in assessing human BAT. To date, FDG-PET is a standard tool used to measure human BAT [121]; however, this option has serious limitations, including the enormous cost of devices, radiation exposure, and acute cold exposure, which make repeated measurements difficult and an impedi- ment in basic and clinical studies. There is therefore an urgent need to establish less invasive and simpler methods for quantitative assessment of human BAT. This would promote dedicated human studies, including longitudinal observations and the development of practical, easy, and effective regimens that can activate and recruit BAT.

\section{CONFLICTS OF INTEREST}

No potential conflict of interest relevant to this article was reported.

\section{ORCID}

Okamatsu-Ogura Yuko https://orcid.org/0000-0002-3008-9080

Masayuki Saito https://orcid.org/0000-0002-3058-3003

\section{FUNDING}

This study was supported by Grant-in-Aid for Scientific Research from the Ministry of Education, Culture, Sports, Science and Technology of Japan, 17K08118 to Okamatsu-Ogura Yuko, and 16K15485, 18K11013 to Masayuki Saito.

\section{ACKNOWLEDGMENTS}

None

\section{REFERENCES}

1. Cannon B, Nedergaard J. Brown adipose tissue: function and physiological significance. Physiol Rev 2004;84:277-359.

2. Saito M, Okamatsu-Ogura Y, Matsushita M, Watanabe K, Yoneshiro T, Nio-Kobayashi J, et al. High incidence of metabolically active brown adipose tissue in healthy adult humans: effects of cold exposure and adiposity. Diabetes 2009;58:152631.

3. van Marken Lichtenbelt WD, Vanhommerig JW, Smulders NM, Drossaerts JM, Kemerink GJ, Bouvy ND, et al. Cold-activated brown adipose tissue in healthy men. $\mathrm{N}$ Engl J Med 2009;360:1500-8.

4. Cypess AM, Lehman S, Williams G, Tal I, Rodman D, Goldfine $\mathrm{AB}$, et al. Identification and importance of brown adipose tissue in adult humans. N Engl J Med 2009;360:1509-17.

5. Virtanen KA, Lidell ME, Orava J, Heglind M, Westergren R, 
Niemi T, et al. Functional brown adipose tissue in healthy adults. N Engl J Med 2009;360:1518-25.

6. Saito M. Brown adipose tissue as a regulator of energy expenditure and body fat in humans. Diabetes Metab J 2013;37:229.

7. Carpentier AC, Blondin DP, Virtanen KA, Richard D, Haman F, Turcotte EE. Brown adipose tissue energy metabolism in humans. Front Endocrinol (Lausanne) 2018;9:447.

8. Saito M, Matsushita M, Yoneshiro T, Okamatsu-Ogura Y. Brown adipose tissue, diet-induced thermogenesis, and thermogenic food ingredients: from mice to men. Front Endocrinol (Lausanne) 2020;11:222.

9. Ikeda K, Yamada T. UCP1 dependent and independent thermogenesis in brown and beige adipocytes. Front Endocrinol (Lausanne) 2020;11:498.

10. Kajimura S, Spiegelman BM, Seale P. Brown and beige fat: physiological roles beyond heat generation. Cell Metab 2015; 22:546-59.

11. Villarroya J, Cereijo R, Gavalda-Navarro A, Peyrou M, Giralt $\mathrm{M}$, Villarroya F. New insights into the secretory functions of brown adipose tissue. J Endocrinol 2019;243:R19-27.

12. Enerback S, Jacobsson A, Simpson EM, Guerra C, Yamashita H, Harper ME, et al. Mice lacking mitochondrial uncoupling protein are cold-sensitive but not obese. Nature 1997;387:904.

13. Shabalina IG, Hoeks J, Kramarova TV, Schrauwen P, Cannon B, Nedergaard J. Cold tolerance of UCP1-ablated mice: a skeletal muscle mitochondria switch toward lipid oxidation with marked UCP3 up-regulation not associated with increased basal, fatty acid- or ROS-induced uncoupling or enhanced GDP effects. Biochim Biophys Acta 2010;1797:968-80.

14. Bal NC, Singh S, Reis FCG, Maurya SK, Pani S, Rowland LA, et al. Both brown adipose tissue and skeletal muscle thermogenesis processes are activated during mild to severe cold adaptation in mice. J Biol Chem 2017;292:16616-25.

15. Bukowiecki LJ, Geloen A, Collet AJ. Proliferation and differentiation of brown adipocytes from interstitial cells during cold acclimation. Am J Physiol 1986;250:C880-7.

16. Fukano K, Okamatsu-Ogura Y, Tsubota A, Nio-Kobayashi J, Kimura K. Cold exposure induces proliferation of mature brown adipocyte in a $\beta 3$-adrenergic receptor-mediated pathway. PLoS One 2016;11:e0166579.

17. Okamatsu-Ogura Y, Fukano K, Tsubota A, Uozumi A, Terao A, Kimura K, et al. Thermogenic ability of uncoupling protein 1 in beige adipocytes in mice. PLoS One 2013;8:e84229.
18. Shabalina IG, Petrovic N, de Jong JM, Kalinovich AV, Cannon B, Nedergaard J. UCP1 in brite/beige adipose tissue mitochondria is functionally thermogenic. Cell Rep 2013;5:1196203.

19. Pant M, Bal NC, Periasamy M. Sarcolipin: a key thermogenic and metabolic regulator in skeletal muscle. Trends Endocrinol Metab 2016;27:881-92.

20. Ikeda K, Kang Q, Yoneshiro T, Camporez JP, Maki H, Homma $\mathrm{M}$, et al. UCP1-independent signaling involving SERCA2bmediated calcium cycling regulates beige fat thermogenesis and systemic glucose homeostasis. Nat Med 2017;23:1454-65.

21. Kazak L, Chouchani ET, Jedrychowski MP, Erickson BK, Shinoda K, Cohen P, et al. A creatine-driven substrate cycle enhances energy expenditure and thermogenesis in beige fat. Cell 2015;163:643-55.

22. Granneman JG, Burnazi M, Zhu Z, Schwamb LA. White adipose tissue contributes to UCP1-independent thermogenesis. Am J Physiol Endocrinol Metab 2003;285:E1230-6.

23. Lidell ME, Betz MJ, Dahlqvist Leinhard O, Heglind M, Elander L, Slawik M, et al. Evidence for two types of brown adipose tissue in humans. Nat Med 2013;19:631-4.

24. Shinoda K, Luijten IH, Hasegawa Y, Hong H, Sonne SB, Kim $\mathrm{M}$, et al. Genetic and functional characterization of clonally derived adult human brown adipocytes. Nat Med 2015;21: 389-94.

25. Fedorenko A, Lishko PV, Kirichok Y. Mechanism of fatty-acid-dependent UCP1 uncoupling in brown fat mitochondria. Cell 2012;151:400-13.

26. Divakaruni AS, Humphrey DM, Brand MD. Fatty acids change the conformation of uncoupling protein 1 (UCP1). J Biol Chem 2012;287:36845-53.

27. Labbe SM, Caron A, Bakan I, Laplante M, Carpentier AC, Lecomte R, et al. In vivo measurement of energy substrate contribution to cold-induced brown adipose tissue thermogenesis. FASEB J 2015;29:2046-58.

28. Blondin DP, Frisch F, Phoenix S, Guerin B, Turcotte EE, Haman F, et al. Inhibition of intracellular triglyceride lipolysis suppresses cold-induced brown adipose tissue metabolism and increases shivering in humans. Cell Metab 2017;25:43847.

29. Schreiber R, Diwoky C, Schoiswohl G, Feiler U, Wongsiriroj $\mathrm{N}$, Abdellatif $\mathrm{M}$, et al. Cold-induced thermogenesis depends on ATGL-mediated lipolysis in cardiac muscle, but not brown adipose tissue. Cell Metab 2017;26:753-63.

30. Shin H, Ma Y, Chanturiya T, Cao Q, Wang Y, Kadegowda 
AKG, et al. Lipolysis in brown adipocytes is not essential for cold-induced thermogenesis in mice. Cell Metab 2017;26:76477.

31. Chitraju C, Fischer AW, Farese RV Jr, Walther TC. Lipid droplets in brown adipose tissue are dispensable for cold-induced thermogenesis. Cell Rep 2020;33:108348.

32. Okamatsu-Ogura Y, Kuroda M, Tsutsumi R, Tsubota A, Saito M, Kimura K, et al. UCP1-dependent and UCP1-independent metabolic changes induced by acute cold exposure in brown adipose tissue of mice. Metabolism 2020;113:154396.

33. Mills EL, Pierce KA, Jedrychowski MP, Garrity R, Winther S, Vidoni S, et al. Accumulation of succinate controls activation of adipose tissue thermogenesis. Nature 2018;560:102-6.

34. Chouchani ET, Kazak L, Jedrychowski MP, Lu GZ, Erickson BK, Szpyt J, et al. Mitochondrial ROS regulate thermogenic energy expenditure and sulfenylation of UCP1. Nature 2016; 532:112-6.

35. Vallerand AL, Perusse F, Bukowiecki LJ. Stimulatory effects of cold exposure and cold acclimation on glucose uptake in rat peripheral tissues. Am J Physiol 1990;259:R1043-9.

36. Shimizu Y, Nikami H, Tsukazaki K, Machado UF, Yano H, Seino Y, et al. Increased expression of glucose transporter GLUT-4 in brown adipose tissue of fasted rats after cold exposure. Am J Physiol 1993;264:E890-5.

37. Shimizu Y, Nikami H, Saito M. Sympathetic activation of glucose utilization in brown adipose tissue in rats. J Biochem 1991;110:688-92.

38. Inokuma K, Ogura-Okamatsu Y, Toda C, Kimura K, Yamashita $\mathrm{H}$, Saito M. Uncoupling protein 1 is necessary for norepinephrine-induced glucose utilization in brown adipose tissue. Diabetes 2005;54:1385-91.

39. Winther S, Isidor MS, Basse AL, Skjoldborg N, Cheung A, Quistorff B, et al. Restricting glycolysis impairs brown adipocyte glucose and oxygen consumption. Am J Physiol Endocrinol Metab 2018;314:E214-23.

40. Jeong JH, Chang JS, Jo YH. Intracellular glycolysis in brown adipose tissue is essential for optogenetically induced nonshivering thermogenesis in mice. Sci Rep 2018;8:6672.

41. Lopez-Soriano FJ, Alemany M. Effect of cold-temperature exposure and acclimation on amino acid pool changes and enzyme activities of rat brown adipose tissue. Biochim Biophys Acta 1987;925:265-71.

42. Lu X, Solmonson A, Lodi A, Nowinski SM, Sentandreu E, Riley CL, et al. The early metabolomic response of adipose tissue during acute cold exposure in mice. Sci Rep 2017;7:3455.
43. Ritchie JW, Baird FE, Christie GR, Stewart A, Low SY, Hundal HS, et al. Mechanisms of glutamine transport in rat adipocytes and acute regulation by cell swelling. Cell Physiol Biochem 2001;11:259-70.

44. Lopez-Soriano FJ, Fernandez-Lopez JA, Mampel T, Villarroya F, Iglesias R, Alemany M. Amino acid and glucose uptake by rat brown adipose tissue. Effect of cold-exposure and acclimation. Biochem J 1988;252:843-9.

45. Kowalchuk JM, Curi R, Newsholme EA. Glutamine metabolism in isolated incubated adipocytes of the rat. Biochem J 1988;249:705-8.

46. Xue Y, Petrovic N, Cao R, Larsson O, Lim S, Chen S, et al. Hypoxia-independent angiogenesis in adipose tissues during cold acclimation. Cell Metab 2009;9:99-109.

47. Wise DR, Ward PS, Shay JE, Cross JR, Gruber JJ, Sachdeva $\mathrm{UM}$, et al. Hypoxia promotes isocitrate dehydrogenase-dependent carboxylation of $\alpha$-ketoglutarate to citrate to support cell growth and viability. Proc Natl Acad Sci U S A 2011;108: 19611-6.

48. Metallo CM, Gameiro PA, Bell EL, Mattaini KR, Yang J, Hiller $\mathrm{K}$, et al. Reductive glutamine metabolism by IDH1 mediates lipogenesis under hypoxia. Nature 2011;481:380-4.

49. Yoneshiro T, Wang Q, Tajima K, Matsushita M, Maki H, Igarashi $\mathrm{K}$, et al. BCAA catabolism in brown fat controls energy homeostasis through SLC25A44. Nature 2019;572:614-9.

50. Liu X, Zheng Z, Zhu X, Meng M, Li L, Shen Y, et al. Brown adipose tissue transplantation improves whole-body energy metabolism. Cell Res 2013;23:851-4.

51. Liu X, Wang S, You Y, Meng M, Zheng Z, Dong M, et al. Brown adipose tissue transplantation reverses obesity in ob/ ob Mice. Endocrinology 2015;156:2461-9.

52. Liu X, Rossmeisl M, McClaine J, Riachi M, Harper ME, Kozak LP. Paradoxical resistance to diet-induced obesity in UCP1deficient mice. J Clin Invest 2003;111:399-407.

53. Inokuma K, Okamatsu-Ogura Y, Omachi A, Matsushita Y, Kimura K, Yamashita H, et al. Indispensable role of mitochondrial UCP1 for antiobesity effect of beta3-adrenergic stimulation. Am J Physiol Endocrinol Metab 2006;290:E1014-21.

54. Kontani Y, Wang Y, Kimura K, Inokuma KI, Saito M, SuzukiMiura T, et al. UCP1 deficiency increases susceptibility to dietinduced obesity with age. Aging Cell 2005;4:147-55.

55. Feldmann HM, Golozoubova V, Cannon B, Nedergaard J. UCP1 ablation induces obesity and abolishes diet-induced thermogenesis in mice exempt from thermal stress by living at thermoneutrality. Cell Metab 2009;9:203-9. 
56. Bartelt A, Bruns OT, Reimer R, Hohenberg H, Ittrich H, Peldschus $\mathrm{K}$, et al. Brown adipose tissue activity controls triglyceride clearance. Nat Med 2011;17:200-5.

57. Berbee JF, Boon MR, Khedoe PP, Bartelt A, Schlein C, Worthmann A, et al. Brown fat activation reduces hypercholesterolaemia and protects from atherosclerosis development. Nat Commun 2015;6:6356.

58. Bartelt A, John C, Schaltenberg N, Berbee JFP, Worthmann A, Cherradi $\mathrm{ML}$, et al. Thermogenic adipocytes promote HDL turnover and reverse cholesterol transport. Nat Commun 2017;8:15010.

59. Bukowiecki LJ. Energy balance and diabetes. The effects of cold exposure, exercise training, and diet composition on glucose tolerance and glucose metabolism in rat peripheral tissues. Can J Physiol Pharmacol 1989;67:382-93.

60. Gunawardana SC, Piston DW. Reversal of type 1 diabetes in mice by brown adipose tissue transplant. Diabetes 2012;61: 674-82.

61. Stanford KI, Middelbeek RJ, Townsend KL, An D, Nygaard $\mathrm{EB}$, Hitchcox KM, et al. Brown adipose tissue regulates glucose homeostasis and insulin sensitivity. J Clin Invest 2013; 123:215-23.

62. Carson C, Macias-Velasco JF, Gunawardana S, Miranda MA, Oyama S, St Pierre CL, et al. Brown adipose expansion and remission of glycemic dysfunction in obese SM/J mice. Cell Rep 2020;33:108237.

63. Nishio M, Yoneshiro T, Nakahara M, Suzuki S, Saeki K, Hasegawa M, et al. Production of functional classical brown adipocytes from human pluripotent stem cells using specific hemopoietin cocktail without gene transfer. Cell Metab 2012; 16:394-406.

64. Asano A, Morimatsu M, Nikami H, Yoshida T, Saito M. Adrenergic activation of vascular endothelial growth factor mRNA expression in rat brown adipose tissue: implication in cold-induced angiogenesis. Biochem J 1997;328:179-83.

65. Nechad M, Ruka E, Thibault J. Production of nerve growth factor by brown fat in culture: relation with the in vivo developmental stage of the tissue. Comp Biochem Physiol Comp Physiol 1994;107:381-8.

66. Rosell M, Kaforou M, Frontini A, Okolo A, Chan YW, Nikolopoulou E, et al. Brown and white adipose tissues: intrinsic differences in gene expression and response to cold exposure in mice. Am J Physiol Endocrinol Metab 2014;306:E945-64.

67. Qing H, Desrouleaux R, Israni-Winger K, Mineur YS, Fogelman $\mathrm{N}$, Zhang $\mathrm{C}$, et al. Origin and function of stress-induced
IL-6 in murine models. Cell 2020;182:1660.

68. Fisher FM, Maratos-Flier E. Understanding the physiology of FGF21. Annu Rev Physiol 2016;78:223-41.

69. Chartoumpekis DV, Habeos IG, Ziros PG, Psyrogiannis AI, Kyriazopoulou VE, Papavassiliou AG. Brown adipose tissue responds to cold and adrenergic stimulation by induction of FGF21. Mol Med 2011;17:736-40.

70. Hondares E, Iglesias R, Giralt A, Gonzalez FJ, Giralt M, Mampel T, et al. Thermogenic activation induces FGF21 expression and release in brown adipose tissue. J Biol Chem 2011;286: 12983-90.

71. Hanssen MJ, Broeders E, Samms RJ, Vosselman MJ, van der Lans AA, Cheng CC, et al. Serum FGF21 levels are associated with brown adipose tissue activity in humans. Sci Rep 2015;5: 10275.

72. Samms RJ, Smith DP, Cheng CC, Antonellis PP, Perfield JW 2nd, Kharitonenkov A, et al. Discrete aspects of FGF21 in vivo pharmacology do not require UCP1. Cell Rep 2015;11:991-9.

73. Veniant MM, Sivits G, Helmering J, Komorowski R, Lee J, Fan W, et al. Pharmacologic effects of FGF21 are independent of the "Browning" of white adipose tissue. Cell Metab 2015;21: 731-8.

74. Fisher FM, Kleiner S, Douris N, Fox EC, Mepani RJ, Verdeguer F, et al. FGF21 regulates PGC-1 $\alpha$ and browning of white adipose tissues in adaptive thermogenesis. Genes Dev 2012;26: 271-81.

75. Keipert S, Lutter D, Schroeder BO, Brandt D, Stahlman M, Schwarzmayr T, et al. Endogenous FGF21-signaling controls paradoxical obesity resistance of UCP1-deficient mice. Nat Commun 2020;11:624.

76. Ruan CC, Kong LR, Chen XH, Ma Y, Pan XX, Zhang ZB, et al. $\mathrm{A} 2 \mathrm{~A}$ receptor activation attenuates hypertensive cardiac remodeling via promoting brown adipose tissue-derived FGF21. Cell Metab 2018;28:476-89.

77. Thomou T, Mori MA, Dreyfuss JM, Konishi M, Sakaguchi M, Wolfrum C, et al. Adipose-derived circulating miRNAs regulate gene expression in other tissues. Nature 2017;542:450-5.

78. Chen Y, Buyel JJ, Hanssen MJ, Siegel F, Pan R, Naumann J, et al. Exosomal microRNA miR-92a concentration in serum reflects human brown fat activity. Nat Commun 2016;7:11420.

79. Okamatsu-Ogura Y, Matsushita M, Bariuan JV, Nagaya K, Tsubota A, Saito M. Association of circulating exosomal miR122 levels with BAT activity in healthy humans. Sci Rep 2019; 9:13243.

80. Simcox J, Geoghegan G, Maschek JA, Bensard CL, Pasquali M, 
Miao R, et al. Global analysis of plasma lipids identifies liverderived acylcarnitines as a fuel source for brown fat thermogenesis. Cell Metab 2017;26:509-22.

81. Lynes MD, Leiria LO, Lundh M, Bartelt A, Shamsi F, Huang TL, et al. The cold-induced lipokine 12,13-diHOME promotes fatty acid transport into brown adipose tissue. Nat Med 2017; 23:631-7.

82. Stanford KI, Lynes MD, Takahashi H, Baer LA, Arts PJ, May FJ, et al. 12,13-diHOME: an exercise-induced lipokine that increases skeletal muscle fatty acid uptake. Cell Metab 2018;27: 1111-20.

83. Wang GX, Zhao XY, Meng ZX, Kern M, Dietrich A, Chen Z, et al. The brown fat-enriched secreted factor $\mathrm{Nrg} 4$ preserves metabolic homeostasis through attenuation of hepatic lipogenesis. Nat Med 2014;20:1436-43.

84. Kong X, Yao T, Zhou P, Kazak L, Tenen D, Lyubetskaya A, et al. Brown adipose tissue controls skeletal muscle function via the secretion of myostatin. Cell Metab 2018;28:631-43.

85. Lee MW, Lee M, Oh KJ. Adipose tissue-derived signatures for obesity and type 2 diabetes: adipokines, batokines and MicroRNAs. J Clin Med 2019;8:854.

86. Scheele C, Wolfrum C. Brown adipose crosstalk in tissue plasticity and human metabolism. Endocr Rev 2020;41:53-65.

87. Okamatsu-Ogura Y, Uozumi A, Toda C, Kimura K, Yamashita $\mathrm{H}$, Saito M. Uncoupling protein 1 contributes to fat-reducing effect of leptin. Obes Res Clin Pract 2007;1:223-90.

88. Yamada T, Katagiri H, Ishigaki Y, Ogihara T, Imai J, Uno K, et al. Signals from intra-abdominal fat modulate insulin and leptin sensitivity through different mechanisms: neuronal involvement in food-intake regulation. Cell Metab 2006;3:2239.

89. Ryu V, Garretson JT, Liu Y, Vaughan CH, Bartness TJ. Brown adipose tissue has sympathetic-sensory feedback circuits. J Neurosci 2015;35:2181-90.

90. Yoneshiro T, Aita S, Matsushita M, Kameya T, Nakada K, Kawai Y, et al. Brown adipose tissue, whole-body energy expenditure, and thermogenesis in healthy adult men. Obesity (Silver Spring) 2011;19:13-6.

91. Orava J, Nuutila P, Lidell ME, Oikonen V, Noponen T, Viljanen T, et al. Different metabolic responses of human brown adipose tissue to activation by cold and insulin. Cell Metab 2011;14:272-9.

92. Ouellet V, Labbe SM, Blondin DP, Phoenix S, Guerin B, Haman F, et al. Brown adipose tissue oxidative metabolism contributes to energy expenditure during acute cold exposure in humans. J Clin Invest 2012;122:545-52.

93. Chen KY, Brychta RJ, Linderman JD, Smith S, Courville A, Dieckmann W, et al. Brown fat activation mediates cold-induced thermogenesis in adult humans in response to a mild decrease in ambient temperature. J Clin Endocrinol Metab 2013;98:E1218-23.

94. Din MU, Saari T, Raiko J, Kudomi N, Maurer SF, Lahesmaa M, et al. Postprandial oxidative metabolism of human brown fat indicates thermogenesis. Cell Metab 2018;28:207-16.

95. Hibi M, Oishi S, Matsushita M, Yoneshiro T, Yamaguchi T, Usui $\mathrm{C}$, et al. Brown adipose tissue is involved in diet-induced thermogenesis and whole-body fat utilization in healthy humans. Int J Obes (Lond) 2016;40:1655-61.

96. Esterbauer H, Oberkofler H, Liu YM, Breban D, Hell E, Krempler F, et al. Uncoupling protein-1 mRNA expression in obese human subjects: the role of sequence variations at the uncoupling protein-1 gene locus. J Lipid Res 1998;39:834-44.

97. Nagai N, Sakane N, Fujishita A, Fujiwara R, Kimura T, Kotani $\mathrm{K}$, et al. The $-3826 \mathrm{~A} \rightarrow \mathrm{G}$ variant of the uncoupling protein-1 gene diminishes thermogenesis during acute cold exposure in healthy children. Obes Res Clin Pract 2007;1:I-II.

98. Nagai N, Sakane N, Ueno LM, Hamada T, Moritani T. The $-3826 \mathrm{~A} \rightarrow \mathrm{G}$ variant of the uncoupling protein-1 gene diminishes postprandial thermogenesis after a high fat meal in healthy boys. J Clin Endocrinol Metab 2003;88:5661-7.

99. Muzik O, Mangner TJ, Leonard WR, Kumar A, Janisse J, Granneman JG. 15O PET measurement of blood flow and oxygen consumption in cold-activated human brown fat. J Nucl Med 2013;54:523-31.

100. Leitner BP, Huang S, Brychta RJ, Duckworth CJ, Baskin AS, McGehee $S$, et al. Mapping of human brown adipose tissue in lean and obese young men. Proc Natl Acad Sci U S A 2017; 114:8649-54.

101. Yoneshiro T, Aita S, Matsushita M, Okamatsu-Ogura Y, Kameya T, Kawai Y, et al. Age-related decrease in cold-activated brown adipose tissue and accumulation of body fat in healthy humans. Obesity (Silver Spring) 2011;19:1755-60.

102. Ouellet V, Routhier-Labadie A, Bellemare W, Lakhal-Chaieb L, Turcotte E, Carpentier AC, et al. Outdoor temperature, age, sex, body mass index, and diabetic status determine the prevalence, mass, and glucose-uptake activity of 18F-FDG-detected BAT in humans. J Clin Endocrinol Metab 2011;96:192-9.

103. Zhang Q, Ye H, Miao Q, Zhang Z, Wang Y, Zhu X, et al. Differences in the metabolic status of healthy adults with and without active brown adipose tissue. Wien Klin Wochenschr 
2013;125:687-95.

104. Matsushita M, Yoneshiro T, Aita S, Kameya T, Sugie H, Saito M. Impact of brown adipose tissue on body fatness and glucose metabolism in healthy humans. Int J Obes (Lond) 2014; 38:812-7.

105. Yoneshiro T, Aita S, Matsushita M, Kayahara T, Kameya T, Kawai Y, et al. Recruited brown adipose tissue as an antiobesity agent in humans. J Clin Invest 2013;123:3404-8.

106. van der Lans AA, Hoeks J, Brans B, Vijgen GH, Visser MG, Vosselman MJ, et al. Cold acclimation recruits human brown fat and increases nonshivering thermogenesis. J Clin Invest 2013;123:3395-403.

107. Hanssen MJ, van der Lans AA, Brans B, Hoeks J, Jardon KM, Schaart G, et al. Short-term cold acclimation recruits brown adipose tissue in obese humans. Diabetes 2016;65:1179-89.

108. Chondronikola M, Volpi E, Borsheim E, Porter C, Annamalai $\mathrm{P}$, Enerback $\mathrm{S}$, et al. Brown adipose tissue improves wholebody glucose homeostasis and insulin sensitivity in humans. Diabetes 2014;63:4089-99.

109. Lee P, Smith S, Linderman J, Courville AB, Brychta RJ, Dieckmann W, et al. Temperature-acclimated brown adipose tissue modulates insulin sensitivity in humans. Diabetes 2014;63: 3686-98.

110. Iwen KA, Backhaus J, Cassens M, Waltl M, Hedesan OC, Merkel M, et al. Cold-induced brown adipose tissue activity alters plasma fatty acids and improves glucose metabolism in men. J Clin Endocrinol Metab 2017;102:4226-34.

111. Hanssen MJ, Hoeks J, Brans B, van der Lans AA, Schaart G, van den Driessche JJ, et al. Short-term cold acclimation improves insulin sensitivity in patients with type 2 diabetes mellitus. Nat Med 2015;21:863-5.

112. Newgard CB, An J, Bain JR, Muehlbauer MJ, Stevens RD, Lien LF, et al. A branched-chain amino acid-related metabolic signature that differentiates obese and lean humans and contrib- utes to insulin resistance. Cell Metab 2009;9:311-26.

113. Tremblay F, Lavigne C, Jacques H, Marette A. Role of dietary proteins and amino acids in the pathogenesis of insulin resistance. Annu Rev Nutr 2007;27:293-310.

114. Becher T, Palanisamy S, Kramer DJ, Eljalby M, Marx SJ, Wibmer AG, et al. Brown adipose tissue is associated with cardiometabolic health. Nat Med 2021;27:58-65.

115. Raiko J, Orava J, Savisto N, Virtanen KA. High brown fat activity correlates with cardiovascular risk factor levels crosssectionally and subclinical atherosclerosis at 5-year follow-up. Arterioscler Thromb Vasc Biol 2020;40:1289-95.

116. Giordano A, Frontini A, Cinti S. Convertible visceral fat as a therapeutic target to curb obesity. Nat Rev Drug Discov 2016; 15:405-24.

117. Yoneshiro T, Matsushita M, Saito M. Translational aspects of brown fat activation by food-derived stimulants. Handb Exp Pharmacol 2019;251:359-79.

118. Koza RA, Hohmann SM, Guerra C, Rossmeisl M, Kozak LP. Synergistic gene interactions control the induction of the mitochondrial uncoupling protein (Ucp1) gene in white fat tissue. J Biol Chem 2000;275:34486-92.

119. Xue B, Rim JS, Hogan JC, Coulter AA, Koza RA, Kozak LP. Genetic variability affects the development of brown adipocytes in white fat but not in interscapular brown fat. J Lipid Res 2007;48:41-51.

120. Yoneshiro T, Ogawa T, Okamoto N, Matsushita M, Aita S, Kameya T, et al. Impact of UCP1 and $\beta 3$ AR gene polymorphisms on age-related changes in brown adipose tissue and adiposity in humans. Int J Obes (Lond) 2013;37:993-8.

121. Chen KY, Cypess AM, Laughlin MR, Haft CR, Hu HH, Bredella MA, et al. Brown adipose reporting criteria in imaging STudies (BARCIST 1.0): recommendations for standardized FDG-PET/CT experiments in humans. Cell Metab 2016;24: 210-22. 